${ }^{1}$ Department of Oral and Maxillofacial Surgery, School of Dentistry, University of Pernambuco, Recife Pernambuco, Brazil.
*Corresponding author: Ricardo José de Holanda Vasconcellos Email: ricardo.holanda@upe.br

Received for publication: June 9, 2020 Accepted: December 18, 2020

\section{Feasibility of} three-dimensional CT reconstruction in assessing mandibular fractures: a survey among oral surgeons and dental maxillofacial radiologists

\author{
Victor Nogueira Moura' ${ }^{1}$, Emerson Nogueira' (iD, \\ Ewerton Daniel Rocha Rodrigues ${ }^{1, \star}$ (iD), Caio César \\ Gonçalves Silva ${ }^{1}$ (D), Ricardo José de Holanda \\ Vasconcellos ${ }^{1}$ (iD)
}

Aim: Evaluation of the reliability of 3D computed tomography (3D-CT) in the diagnosis of mandibular fractures. Methods: A cross-sectional, quantitative and qualitative study was carried out, through the application of a questionnaire for 70 professionals in the area of Oral and Maxillofacial Surgery and Radiology. 3D-CT images of mandibular fractures were delivered to the interviewees along with a questionnaire. Participants answered about the number of traces, the region and the type of fracture. The correct diagnosis, that is, the expected answer, was based on the reports of a specialist in oral and maxillofacial radiology after viewing the images in the axial, sagittal and coronal sections. The resulting data from the interviewees was compared with the expected answer and then, the data was analyzed statistically. Results: In the sample $56.9 \%$ were between 22 and 30 years old, $52.8 \%$ were oral and maxillofacial surgeons (OMF), $34.7 \%$ were residents in OMF surgery and $12.5 \%$ OMF radiologists. Each professional answered 15 questions (related to five patients) and 50.8\% of the total of these was answered correctly. Specialists in Oral and Maxillofacial Surgery and Traumatology correctly answered 53.9\%. Interviewees with experience between 6 and 10 years correctly answered 58.2\%. In identifying fracture traces, $46.1 \%$ of the questions were answered correctly. In terms of location, 5.6\% of interviewees answered wrongly while $14.2 \%$ answered wrongly regarding classification. Conclusion: 3D computed tomography did 
not prove to be a reliable image for diagnosing mandibular fractures when used alone. This made necessary an association with axial, sagittal and coronal tomographic sections.

Keywords: Tomography, X-ray computed. Imaging, three-dimensional. Diagnostic imaging. Mandibular fractures.

\section{Introduction}

In recent years, computed tomography (CT) has enabled better accuracy assessment of face fractures. It boosted and made imaging examination to present the best details, enabling the surgeon to view structures in three dimensions ${ }^{1,2}$. Fracture traces, location, extension and displacement of fragments are evaluated in sections without image overlap?

Two-dimensionally (2D) or three-dimensional (3D) reconstructed images can be obtained from original CT data, which allow indirect reconstructions in any desired plane $^{3}$. 3D tomographic reconstructions have the advantage of helping communication between professionals, and between professionals and patients, however, according to some authors, they are not reliable in the diagnosis of facial fractures, especially those involving the middle third of the face, due to overlapping of images, artifacts, and the limitation in viewing details ${ }^{4}$.

Some studies ${ }^{3-5}$ suggest the utility of three-dimensional computed tomography technologies for preoperative and intraoperative decision-making, but it has not yet been determined to which level three-dimensional computed tomography (3D-CT) is a useful tool for diagnosing the maxillofacial trauma. Although some professionals have high confidence in 3D-CT in the diagnosis of some facial fractures.

Therefore, the objective of this research was to assess the feasibility of 3D-CT reconstructed images to diagnose mandibular fractures and related factors.

\section{Materials and Methods}

A cross-sectional, quantitative and qualitative study was carried out through the application of a printed questionnaire. This questionnaire was developed specifically for this study by 2 Maxillofacial Surgeons professors of the discipline of Surgery at the University of Pernambuco. The questions asked were reviewed by the radiologist responsible for preparing the answers. All interviewees were informed about the content of the research and signed an informed consent form. The research started after approval by the Research Ethics Committee of Universidade de Pernambuco (UPE), with the CAAE registration: 91652217.3.0000.5207.

Residents, oral and maxilofacial surgeons, as well as oral and maxillofacial radiologists working in the state of Pernambuco (Brazil) participated in the research. Corresponding to a total of 70 participants. The inclusion criteria were: 1) be Oral and Maxillofacial (OMF) surgeon, OMF resident, OMF radiologist and; 2) be active in their professional área. Participants who did not answer the questionnaire completely were excluded. Sampling was through spontaneous demand from professionals who agreed to participate in this study. 
The examinations were performed on young patients (ages ranging from 20 to 36 years) who were victims of facial trauma (motorcycle accident, car accident and physical aggression) treated at a trauma hospital. Computed tomography (CT) was performed using he SOMATOM Definition AS, Siemens (34 chanels). The slice thickness used in this study was $3 \mathrm{~mm}$, the pitch was 1.2 and scan area diameter was $30 \mathrm{~cm}$.

Ten clinical cases of mandible fracture were randomly selected the Excell's randbetween function. From these cases, the printing of 6 images in 3D reconstruction was standardized (frontal, axial, 3/4 right, 3/4 left, right profile, left profile) (Figure 1). The images were printed on photographic paper at $29.7 \times 42.2 \mathrm{~cm}$.

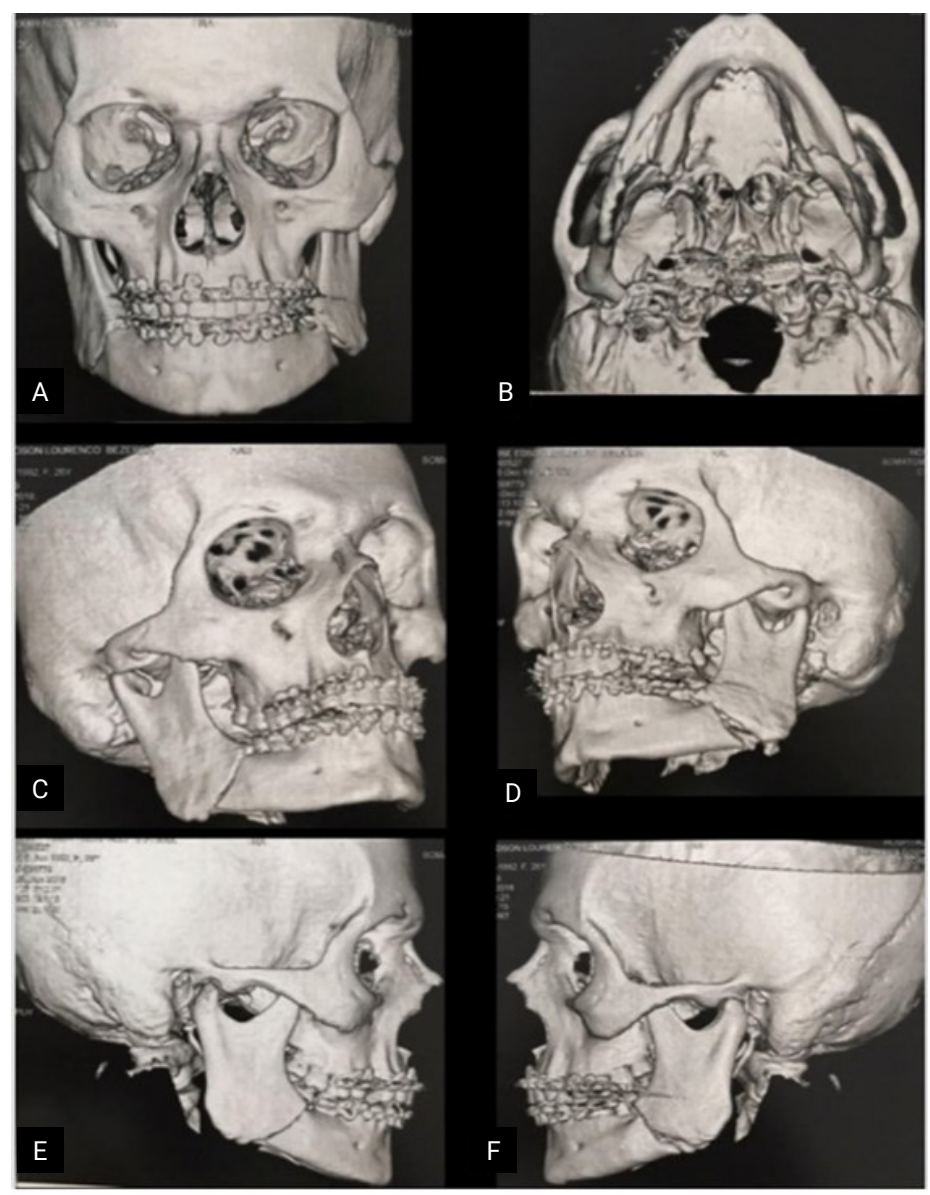

Figure 1. Tomography of a patient in 3D reconstruction in: A) frontal view, B) axial, C) $3 / 4$ right, D) $3 / 4$ left, E) right profile and F) left profile of one of the evaluated clinical cases.

Each participant received a questionnaire containing 3D reconstructed tomographic images representative of five random clinical cases. For each case, the volunteer should have answered the following questions. The questionnaires were randomized using the RANDOM.ORG program, and applied in hospitals and radiological clinics, where each interviewee had 30 minutes to answer the questions. 
The questions were:

A. How many fracture traces can you see in the image? $(1,2,3,4,5$ or more than 5$)$

B. At which region the fractures are located? (condyle, branch, angle, body, parasymphysis, symphysis, dentoalveolar)

C. What is the type of fracture? (simple, complex, incomplete, comminutive, favorable, unfavorable)

In each case the second and third questions could have more than one correct answer. A specialist in oral and maxillofacial radiology gave the correct answer to these questions after analyzing the cases, but with the images in coronal, axial and sagittal section. The radiologist answered the same questions ten days ago to assess the intra-examiner efficiency. It was considered as correct the one answered correctly in its entirety; partially correct when the question was answered incompletely correct; incorrect when neither of the alternatives had been filled out correctly or the candidate did not answer. In addition, demographic data such as age, education and time since graduation were collected from the interviewees. Residents were asked about time since undergraduation. For specialists, the time since graduation.

Data was analyzed descriptively using absolute and percentage frequencies for categorical variables and measures: average, standard deviation, median, minimum and maximum values for numerical variables (age and number of questions: correct, partially correct and incorrect). To assess the association between two categorical variables, the Pearson's chi-squared test was used, or the Fisher's exact test when the condition for using the chi-squared test was not verified. Mann-Whitney test was used to compare the groups for the numerical variables. The choice for the Mann-Whitney test was due to the absence of normality of data, a condition verified through the Shapiro-Wilk test. The margin of error used in deciding the statistical tests was 5\%. The data was entered into EXCEL spreadsheet and the program used to perform the statistical analysis was IBM SPSS version 23.0 (IBM Corporation, Sommers, NY, USA) for Windows (Microsoft Corporation, Redmond, WA, USA).

\section{Results}

Seventy professionals were interviewed and Table 1 presents the results of the sample's demographic profile, 44 male and 26 female. This table highlights that: the average age in the total group was 31.86 years and the median 30.00 years; the age group of 22 to 30 years old was the most prevalent with $56.9 \%$ and the remaining $43.1 \%$ were 31 years old or more. A little more than half $(52.8 \%)$ of the participants were OMF surgeons, followed by $34.7 \%$ who were OMF residents and the remaining $12.5 \%$ were radiologists. The most prevalent "time since graduation" time corresponded to those who were up to 2 years after graduation $(41.4 \%)$ and the other time ranges had percentages ranging from $18.6 \%$ to $21.4 \%$. 
Table 1. Demographic profile evaluation.

\begin{tabular}{lc}
\hline Variable & Total Group \\
\hline Age: Average \pm DP (Median) & $31.86 \pm 9.10(30.00)$ \\
\hline Age range: $\mathrm{n}(\%)^{(1)}$ & $33(56.9)$ \\
\hline 22 to 30 & $25(43.1)$ \\
\hline 31 or more & $58(100.0)$ \\
\hline TOTAL & $38(52.8)$ \\
\hline Specialty: $\mathrm{n}(\%)$ & $25(34.7)$ \\
\hline OMF surgeons & $9(12.5)$ \\
\hline OMF Residents & \\
\hline OMF Radiologists & $29(41.4)$ \\
\hline Formation time (in years): $\mathrm{n}(\%)(2)$ & $13(18.6)$ \\
\hline Until 2 & $15(21.4)$ \\
\hline 3 to 5 & $13(18.6)$ \\
\hline 6 to 10 & $70(100.0)$ \\
\hline More than 10 & \\
\hline TOTAL &
\end{tabular}

(1) For 14 respondents age was not informed.

(2) For two respondents the time since graduation was not informed.

General assessments (that is, regardless of formation) corresponding to the answers given to all questions are shown in table 2. There was an average of 7.63 questions answered in their entirety. For questions with more than one answer, an average of 3.69 partial hits was observed. And there was an average of 3.68 errors in total.

Table 2. Statistics on the number of correct, partially correct and incorrect answers from the 15 questions presented.

\begin{tabular}{lcc}
\hline Variable & Statistics & Total Group \\
\hline \multirow{3}{*}{ Correct Answers in Total } & Average \pm DP & $7.63 \pm 2.80$ \\
\cline { 2 - 3 } & Median & 7.00 \\
\cline { 2 - 3 } & Minimum & 3.00 \\
\cline { 2 - 3 } Partially Correct Answers in Total & Maximum & 14.00 \\
\cline { 2 - 3 } & Average \pm DP & $3.69 \pm 1.42$ \\
\cline { 2 - 3 } & Median & 4.00 \\
\hline \multirow{2}{*}{ Incorrect Answers in Total } & Minimum & 0.00 \\
\cline { 2 - 3 } & Maximum & 7.00 \\
\cline { 2 - 3 } & Average \pm DP & $3.68 \pm 1.90$ \\
\hline & Median & 4.00 \\
\hline
\end{tabular}

Table 3 shows the answers to each question, together with the number of correct answers according to each specialty and formation, while Table 4 shows the results based on formation time. 
Table 3. Evaluation of the total of 15 questions and blocks according to specialty

\begin{tabular}{|c|c|c|c|c|c|c|c|c|c|}
\hline \multirow{3}{*}{ Variable } & \multicolumn{8}{|c|}{ Speciality } & \multirow{3}{*}{$\mathrm{p}$ Value } \\
\hline & \multicolumn{2}{|c|}{ Specialist } & \multicolumn{2}{|c|}{ Resident } & \multicolumn{2}{|c|}{ Radiologist } & \multicolumn{2}{|c|}{ Total Group } & \\
\hline & $\mathrm{n}$ & $\%$ & $\mathrm{n}$ & $\%$ & $\mathrm{n}$ & $\%$ & $\mathrm{n}$ & $\%$ & \\
\hline \multicolumn{10}{|l|}{ Total Block } \\
\hline Correct & 307 & 53.9 & 181 & 48.3 & 61 & 45.2 & 549 & 50.8 & \multirow{3}{*}{$\mathrm{p}^{(1)}=0.290$} \\
\hline Partially Correct & 134 & 23.5 & 95 & 25.3 & 37 & 27.4 & 266 & 24.6 & \\
\hline Incorrect & 129 & 22.6 & 99 & 26.4 & 37 & 27.4 & 265 & 24.5 & \\
\hline \multicolumn{10}{|c|}{ Questions about fracture trace } \\
\hline Correct & 92 & 48.4 & 53 & 42.4 & 21 & 46.7 & 166 & 46.1 & \multirow{2}{*}{$\mathrm{p}^{(1)}=0.575$} \\
\hline Incorrect & 98 & 51.6 & 72 & 57.6 & 24 & 53.3 & 194 & 53.9 & \\
\hline \multicolumn{10}{|c|}{ Diagnosis of the fractured region } \\
\hline Correct & 93 & 48.9 & 52 & 41.6 & 17 & 37.8 & 162 & 45.0 & \multirow{3}{*}{$\mathrm{p}^{(1)}=0.541$} \\
\hline Partially Correct & 87 & 45.8 & 65 & 52.0 & 26 & 57.8 & 178 & 49.4 & \\
\hline Incorrect & 10 & 5.3 & 8 & 6.4 & 2 & 4.4 & 20 & 5.6 & \\
\hline \multicolumn{10}{|c|}{ Fracture classification } \\
\hline Correct & 122 & 64.2 & 76 & 60.8 & 23 & 51.1 & 221 & 61.4 & \multirow{3}{*}{$\mathrm{p}^{(1)}=0.215$} \\
\hline Partially Correct & 47 & 24.7 & 30 & 24.0 & 11 & 24.4 & 88 & 24.4 & \\
\hline Incorrect & 21 & 11.1 & 19 & 15.2 & 11 & 24.4 & 51 & 14.2 & \\
\hline
\end{tabular}

(*) Significant difference at the level of $5.0 \%$

(1) Through Pearson's Chi-square test.

Table 4. Evaluation of the total of 15 questions and blocks according to the time since graduation

\begin{tabular}{|c|c|c|c|c|c|c|c|c|c|c|c|}
\hline \multirow{3}{*}{ Variable } & \multicolumn{8}{|c|}{ Time since graduation } & & & \multirow{3}{*}{ P Value } \\
\hline & \multicolumn{2}{|c|}{ Until 2} & \multicolumn{2}{|c|}{3 to 5} & \multicolumn{2}{|c|}{6 to 10} & \multicolumn{2}{|c|}{ More than 10} & \multicolumn{2}{|c|}{ Total Group } & \\
\hline & $\mathrm{n}$ & $\%$ & $\mathrm{n}$ & $\%$ & $\mathrm{n}$ & $\%$ & $\mathrm{n}$ & $\%$ & $\mathrm{n}$ & $\%$ & \\
\hline \multicolumn{12}{|l|}{ Total Block } \\
\hline Correct & 218 & 50.1 & 107 & 54.9 & 131 & 58.2 & 83 & 42.6 & 539 & 51.3 & \multirow{3}{*}{$\mathrm{p}^{(1)}=0.048^{x}$} \\
\hline Partially Correct & 107 & 24.6 & 48 & 24.6 & 50 & 22.2 & 54 & 27.7 & 259 & 24.7 & \\
\hline Incorrect & 110 & 25.3 & 40 & 20.5 & 44 & 19.6 & 58 & 29.7 & 252 & 24.0 & \\
\hline \multicolumn{12}{|c|}{ Questions about fracture trace } \\
\hline Correct & 63 & 43.4 & 33 & 50.8 & 42 & 56.0 & 25 & 38.5 & 163 & 46.6 & \multirow{2}{*}{$-p^{(1)}=0.143$} \\
\hline Incorrect & 82 & 56.6 & 32 & 49.2 & 33 & 44.0 & 40 & 61.5 & 187 & 53.4 & \\
\hline \multicolumn{12}{|c|}{ Diagnosis of the fractured region } \\
\hline Correct & 64 & 44.1 & 30 & 46.2 & 39 & 52.0 & 26 & 40.0 & 159 & 45.4 & \multirow{3}{*}{$\mathrm{p}^{(2)}=0.971$} \\
\hline Partially Correct & 73 & 50.3 & 32 & 49.2 & 32 & 42.7 & 36 & 55.4 & 173 & 49.4 & \\
\hline Incorrect & 8 & 5.5 & 3 & 4.6 & 4 & 5.3 & 3 & 4.6 & 18 & 5.1 & \\
\hline \multicolumn{12}{|c|}{ Fracture classification } \\
\hline Correct & 91 & 62.8 & 44 & 67.7 & 50 & 66.7 & 32 & 49.2 & 217 & 62.0 & \multirow{3}{*}{$p^{(1)}=0.137$} \\
\hline Partially Correct & 34 & 23.4 & 16 & 24.6 & 18 & 24.0 & 18 & 27.7 & 86 & 24.6 & \\
\hline Incorrect & 20 & 13.8 & 5 & 7.7 & 7 & 9.3 & 15 & 23.1 & 47 & 13.4 & \\
\hline
\end{tabular}

(*) Significant difference at the level of $5.0 \%$

(1) Through Pearson's Chi-square test.

In the general assessment, professionals who had between 6 to 10 years since graduation, obtained the highest rate of correct answers with $58.2 \%$, while professionals with more than 10 years of experience obtained the lowest rate, $42.6 \%$. In questions 
about fracture traces, the highest rate of correct answer was in the range of 6 to 10 years since graduation and the lowest was with the group that was over 10 years since graduation, with $56.0 \%$ and $38.5 \%$ respectively. In the diagnosis of the fractured region, these groups again obtained the highest and lowest rate of correct answers, with $52.0 \%$ and $40.0 \%$ respectively, however the interviewees with more than 10 years of formation, obtained the highest rate of partial correct answers, with $55.4 \%$ against $42.5 \%$ of candidates with experience between 6 and 10 years. The lowest error rate for this assessment was among respondents with experience between 3 and 5 years and with those with more than 10 years, with $4.5 \%$. In the classification of fractures, the group with experience between 3 and 5 years was the one that got the highest rate of correct answers (67.7\%) while the group with more than 10 years of experience was the one that obtained the lowest rate (49.2\%).

\section{Discussion}

Despite the clinical examination being the most important procedure for the correct diagnosis, radiographic investigation is of great importance, and is consequently important in the elaboration of the treatment plan and in the postoperative follow-up of patients with fractures of the facial bones ${ }^{3,6}$. The management of facial fractures is based on accurate clinical and radiographic diagnosis $s^{3,7}$. Of the 15 questions in our study, an average of 7.63 questions was found to be correct answers in their entirety. That is, there may be a difficulty in interpreting 3D images, leading to possible flaws in the diagnosis and consequently in the surgical planning. The literature says that two-dimensional computed tomography with axial, coronal and sagittal projections is a standard criterion for evaluating facial fractures through images, especially when dealing with orbital fractures ${ }^{8}$. However, 3D tomography has become a useful tool in the diagnosis of facial fractures ${ }^{5,8}$. In conventional radiography, anatomy is represented in two dimensions. Facial injuries usually produce significant edema, which can make the diagnosis of underlying bone lesions difficult ${ }^{9}$.

Fox et al. ${ }^{10}$ reported in their study that 3D-CT scans were interpreted in shorter time and with greater precision when compared to conventional CT scans, in addition, these scans were more accurate when assessing zygomatic fractures. However, they also noted that these exams were less effective than 2D exam in the evaluation of orbital fractures. These findings are in line with what was observed in our study, in which 3D-CT scans were not interpreted correctly, with a percentage of $53.9 \%$ of errors in relation to the number of fractures in the mandible.

In our findings, surgery specialists were the group that obtained the highest percentage of correct answers, when compared to residents and radiologists. These findings can be attributed to the fact that specialists usually have more experience in interpreting these exams than the other two groups, since oral radiologists do not use medical tomography frequently. However, it is important to point out the fact that three interviewees (2 surgery specialists and 1 radiologist) were unable to identify any fractures in one of the patients, which could prevent that patient of being treated. This reinforces the importance and sovereignty of the clinical exam in relation to the complementary exams. 
Saigal et al. ${ }^{4}$ drew attention to the usefulness of 3D imaging in cases of complex facial trauma. According to these authors, visualization in three dimensions helps the complex process in which the surgeon visualizes operational planning ${ }^{4}$. This advantage is also pointed out in the clinical trial by Shah et al. ${ }^{11}$. Perandini, et al. ${ }^{12}$ who reinforce in their articles the importance of 3D-CT in the diagnosis of relatively common conditions such as thrombosis, stenosis of the airways, exophytic cancer and trauma.

Wolf et al. ${ }^{13}$ evaluated whether the diagnostic information by 3D imaging had a significant impact on the decision process in six different classes of surgical indications. These authors observed that tomographic images resulted in significantly more relevant surgical information in dental implant and in cases of maxillofacial surgery, however, the 3D image information did not significantly change the surgical plan based on the 2D examination Wolf et al. ${ }^{13}$. In the study by Kaeppler et al. ${ }^{14}$, the authors sought to determine whether 3D tomography would lead to a change in the treatment of patients with suspected jaw fracture with ambiguous conventional radiographic and clinical signs. It was observed that in $63.2 \%$ of cases, the suspected diagnosis was confirmed and additional fractures were identified in $17.75 \%$ of the evaluated patients ${ }^{14}$.

Regarding the location of fractures, interviewees in this study were able to correctly observe most cases and obtained few errors. Fox et al. ${ }^{10}$ observed that there is a better understanding of the spatial relationships of fractured elements with 3D, as evidenced by their ability to locate the traces. In our study, radiologists obtained the lowest error rate in the location of the fractures when compared to specialists and residents in the current research.

The combination of section thickness, gap between sections and pipe current can influence the quality of the 3D reconstruction. Sales et al. ${ }^{15}$ reported that 3D reconstructions are accurate for the detection of injuries and destruction of bone marrow. However, these authors drew attention to the fact that the quality of the CT scan can be affected by several digitalization configurations, they also reported that axial sections of $0.5 \mathrm{~mm}$ in width and $0.3 \mathrm{~mm}$ reconstruction interval were used to optimize the results ${ }^{16}$. In compliance with the report by Kim et al. ${ }^{17}$, who observed that thinner sections helped to establish more accurate 3D cranial measurements.

During the development of the research, we can observe some limitations. Infinite forms of fracture can exist, and we select only a few cases. Another difficulty observed was that the interviewees analyzed the printed exams, which makes it difficult to manipulate and observe other tomographic sections. For a better analysis, a larger sample is recommended, and perhaps to analyze the mandibular regions in isolation (only condylar fractures, only symphysis fractures ...). Sensitivity and specificity testing would bring a stronger scientific result.

In conclusion, imaging exam is an essential part of diagnosis and treatment planning for maxillofacial trauma. Although 3D reconstruction provides an important global view of the investigated area, in this research, 3D-CT did not prove to be a reliable image in the visualization of the traces of mandibular fractures when used alone, since only half of the interviewees were able to diagnose them correctly. 


\section{References}

1. Shintaku WH, Venturin JS, Azevedo B, Noujeim M. Applications of cone-beam computed tomography in fractures of the maxillofacial complex. Dent Traumatol. 2009;25(4):358-66. doi: 10.1111/j.16009657.2009.00795.x.

2. Bai L, Li L, Su K, Bleyer A, Zhang Y, Ji P. 3D reconstruction images of cone beam computed tomography applied to maxillofacial fractures: A case study and mini review. J Xray Sci Technol. 2018;26(1):115-23. doi: 10.3233/XST-17342.

3. Aydin U, Gormez O, Yildirim D. Cone-beam computed tomography imaging of dentoalveolar and mandibular fractures. Oral Radiol. 2019;(0123456789):23-8. doi: 10.1007/s11282-019-00390-5.

4. Saigal K, Winokur RS, Finden S, Taub D, Pribitkin E. Use of three-dimensional computerized tomography reconstruction in complex facial trauma. Facial Plast Surg. 2005;21(3):214-9. doi: 10.1055/s-2005-922862.

5. Jarrahy R, Vo V, Goenjian HA, Tabit CJ, Katchikian H V, Kumar A, et al. Diagnostic accuracy of maxillofacial trauma two-dimensional and three-dimensional computed tomographic scans: comparison of oral surgeons, head and neck surgeons, plastic surgeons, and neuroradiologists. Plast Reconstr Surg. 2011;127(6):2432-40. doi: 10.1097/PRS.0b013e318213a1fe.

6. Nardi C, Vignoli C, Pietragalla M, Tonelli P, Calistri L, Franchi L, et al. Imaging of mandibular fractures: a pictorial review. Insights Imaging. 2020;11(1). doi: 10.1186/s13244-020-0837-0.

7. Manson PN, Markowitz B, Mirvis S, Dunham M, Yaremchuk M. Toward CT-based facial fracture treatment (Letter). Plast Reconstr Surg. 1990 Oct;86(4):806.

8. Dediol E. The role of three-dimensional computed tomography in evaluating facial trauma. Plast Reconstr Surg 2012;129(2):354-5. doi: 10.1097/PRS.0b013e31823aee2f.

9. Kaur J, Chopra R. Three Dimensional CT Reconstruction for the evaluation and surgical planning of mid face fractures: a 100 case study. J Maxillofac Oral Surg. 2010;9(4):323-8. doi: 10.1007/s12663-010-0137-1.

10. Fox LA, Vannier MW, West OC, Wilson AJ, Baran GA, Pilgram TK. Diagnostic performance of CT, MPR and 3DCT imaging in maxillofacial trauma. Comput Med Imaging Graph. 1995;19(5):385-95. doi: 10.1016/0895-6111(95)00022-4.

11. Shah S, Uppal SK, Mittal RK, Garg R, Saggar K, Dhawan R. Diagnostic tools in maxillofacial fractures: Is there really a need of three-dimensional computed tomography? Indian J Plast Surg. 2016;49(2):225-33. doi: 10.4103/0970-0358.191320.

12. Perandini S, Faccioli N, Zaccarella A, Re T, Mucelli R. The diagnostic contribution of CT volumetric rendering techniques in routine practice. Indian J Radiol Imaging. 2010;20(2):92-7. doi: 10.4103/0971-3026.63043.

13. Wolff C, Mücke T, Wagenpfeil S, Kanatas A, Bissinger O, Deppe H. Do CBCT scans alter surgical treatment plans? Comparison of preoperative surgical diagnosis using panoramic versus cone-beam CT images. J Cranio-Maxillofacial Surg. 2016;44(10):1700-5. doi: 10.1016/j.jcms.2016.07.025.

14. Kaeppler G, Cornelius CP, Ehrenfeld M, Mast G. Diagnostic efficacy of cone-beam computed tomography for mandibular fractures. Oral Surg Oral Med Oral Pathol Oral Radiol. 2013;116(1):98104. doi: 10.1016/j.0000.2013.04.004.

15. Sales MAO, Gaia BF, Perrela A, Cavalcanti MGP. Comparison between multislice and cone-beam computed tomography for the identification of simulated bone lesions using 3D reconstruction. Rev Odonto Cienc. 2013;28(2):47-52.

16. Severo FC, Barbosa GF. Risk factors and success rates associated with orthodontic mini-implants: a literature review. Rev Odonto Cienc. 2015;30(4):200-4. doi: 10.15448/1980-6523.2015.4.15801.

17. Kim DO, Kim HJ, Jung H, Jeong HK, Hong S II, Kim KD. Quantitative evaluation of acquisition parameters in three-dimensional imaging with multidetector computed tomography using human skull phantom. J Digit Imaging. 2002;15 Suppl 1(4):254-7. doi: 10.1007/s10278-002-5054-5. 